\title{
Seven Steps Toward More Transparency in Statistical Practice
}

\author{
Eric-Jan Wagenmakers ${ }^{* 1}$, Alexandra Sarafoglou ${ }^{1}$, Sil Aarts ${ }^{2}$, Casper \\ Albers $^{3}$, Johannes Algermissen ${ }^{4}$, Štěpán Bahník ${ }^{5}$, Noah van Dongen ${ }^{1}$, Rink \\ Hoekstra $^{6}$, David Moreau ${ }^{7}$, Don van Ravenzwaaij ${ }^{8}$, Aljaž Sluga ${ }^{9}$, Franziska \\ Stanke $^{10}$, Jorge Tendeiro ${ }^{11,8}$, and Balazs Aczel ${ }^{12}$ \\ ${ }^{1}$ Department of Psychology, University of Amsterdam, The Netherlands \\ ${ }^{2}$ School for Public Health and Primary Care, Maastricht University, The Netherlands \\ ${ }^{3}$ Heymans Institute of Psychological Research, University of Groningen, The Netherlands \\ ${ }^{4}$ Donders Institute for Brain, Cognition and Behaviour, Radboud University, The Netherlands \\ ${ }^{5}$ Faculty of Business Administration, Prague University of Economics, Czech Republic \\ ${ }^{6}$ Department of Educational Science, University of Groningen, The Netherlands \\ ${ }^{7}$ School of Psychology and Centre for Brain Research, The University of Auckland, New Zealand \\ ${ }^{8}$ Department of Psychology, University of Groningen, The Netherlands \\ ${ }^{9}$ Rotterdam School of Management, Erasmus University Rotterdam, The Netherlands \\ ${ }^{10}$ Department of Psychology, University of Münster, Germany \\ ${ }^{11}$ Office of Research and Academia-Government-Community Collaboration Education and \\ Research Center for Artificial Intelligence and Data Innovation Hiroshima University \\ ${ }^{12}$ Institute of Psychology, ELTE Eotvos Lorand University, Hungary
}

July 8,2021

${ }^{*}$ Correspondence concerning this article should be addressed to: Eric-Jan Wagenmakers, University of Amsterdam, Nieuwe Achtergracht 129 B, 1018 WT Amsterdam, The Netherlands. E-mail may be sent to ej.wagenmakers@gmail.com. 


\begin{abstract}
We argue that statistical practice in the social and behavioral sciences benefits from transparency, a fair acknowledgement of uncertainty, and openness to alternative interpretations. To promote such a practice, we recommend seven concrete statistical procedures: (1) visualizing data; (2) quantifying inferential uncertainty; (3) assessing data preprocessing choices; (4) reporting multiple models; (5) involving multiple analysts; (6) interpreting results modestly; and (7) sharing data and code. We discuss their benefits and limitations, and provide guidelines for adoption. Each of the seven procedures finds inspiration in Merton's ethos of science as reflected in the norms of communalism, universalism, disinterestedness, and organized skepticism. We believe that these ethical considerations -and their statistical consequences- establish common ground among data analysts, despite continuing disagreements about the foundations of statistical inference.
\end{abstract}




\section{INTRODUCTION}

A superficial assessment of the published literature suggests that statisticians rarely agree on anything. Different schools - mostly frequentists, likelihoodists, and Bayesians- have fought one another tooth and nail for decades, debating the meaning of "probability", arguing about the role of prior knowledge, disputing the value of objective vs. subjective analyses, and disagreeing about the primary goal of inference itself: whether researchers should control error rates, update beliefs, or make coherent decisions. Fundamental disagreement exists not only between the different statistical schools, but is also present within the same school. For instance, within the frequentist school there is the perennial debate between those who seek to test hypotheses through $p$-values and those who emphasize estimation through confidence intervals; and within the Bayesian school, Jack Good's claim that there are 46, 656 varieties of Bayesians may prove an underestimate ( ${ }^{1}$; but see ${ }^{2}$ ).

The disagreement also manifests itself in practical application, whenever multiple statisticians and practitioners of statistics find themselves independently analyzing the same data set. Specifically, recent "multiple-analyst" articles show that statisticians rarely used the same analysis, and often drew different conclusions, even for the exact same data set and research question ${ }^{3-7}$. Deep disagreement is also exhibited by contradictory guidelines on $p$ values (e.g., ${ }^{8-13}$ ). Should practitioners avoid the phrase "statistically significant"? Should they lower the $p$-value thresholds, or justify them, or abandon $p$-values altogether? And if p-values are abandoned, what should replace them? With statisticians fighting over these fundamental issues, users of applied statistics may be forgiven for adopting a wait-and-see attitude and carrying on as usual.

In this article, we claim that besides the numerous disputes and outstanding arguments, statisticians might agree on a set of scientific norms. We bring these norms to the fore, as 
we believe that they have considerable relevance for the practice of statistics in the social and behavioural sciences. The norms which we believe should guide statistical practice are communalism, universalism, disinterestedness, and organized skepticism, which are the four scientific norms proposed by Merton (1973) ${ }^{14}$ (originally published in 1942; see the textbox for a detailed overview of the Mertonian norms).

\section{Merton's Ethos of Science}

Merton ${ }^{14}$ proposed that scientific ethos is characterized by the following four norms:

1. Communalism. "The substantive findings of science are a product of social collaboration and are assigned to the community. ( ...) Property rights in science are whittled down to a bare minimum by the rationale of the scientific ethic. $(\cdots)$ The institutional conception of science as part of the public domain is linked with the imperative for communication of findings. Secrecy is the antithesis of this norm; full and open communication its enactment." 14, pp. 273-274

2. Universalism. "truth-claims, whatever their source, are to be subjected to preestablished impersonal criteria: consonant with observation and with previously confirmed knowledge. The acceptance or rejection of claims entering the lists of science is not to depend on the personal or social attributes of their protagonist; his race, nationality, religion, class, and personal qualities are as such irrelevant." 14 , p. 270; italics in original

3. Disinterestedness. "Science, as is the case with professions in general, includes disinterestedness as a basic institutional element. (...) A passion for knowledge, 
idle curiosity, altruistic concern with the benefit to humanity $(\cdots)$ have been attributed to the scientist." 14 , pp. 275-276

4. Organized Skepticism. This "involves a latent questioning of certain bases of established routine, authority, vested procedures and the realm of the "sacred" generally. ( ...) Science which asks questions of fact concerning every phase of nature and society comes into psychological, not logical, conflict with other attitudes toward these same data which have been crystallized and frequently ritualized by other institutions. Most institutions demand unqualified faith; but the institution of science makes scepticism a virtue." 14, p. 264-265; italics in original

In general, when Mertonian norms are carried over to the field of statistics, general themes include the need to be transparent, to acknowledge uncertainty, and to be open to alternative interpretations. As such, the Mertonian norms, although proposed over half a century ago, embody the current aspirations to increase the transparency and reproducibility of science. Critically, the principles behind the Mertonian norms can be translated into concrete statistical practices. A non-exhaustive list of these practices include (1) visualizing data; (2) quantifying inferential uncertainty; (3) assessing data preprocessing choices; (4) reporting multiple models; (5) involving multiple analysts; (6) interpreting results modestly; (7) sharing data and code. We believe that most statisticians would generally endorse these practices ${ }^{15}$, barring reasonable exceptions (e.g., privacy concerns, severe restrictions of time and money). In this article, we will explain these practices in more detail, including their benefits, limitations and guidelines. 


\section{VISUALIZING DATA}

\section{$2.1 \quad$ Description}

By visualizing data, researchers can graphically represent key aspects of the observed data as well as important properties of the statistical model applied.

\section{$2.2 \quad$ Benefits and Examples}

Data visualization is important in all phases of the statistical workflow. In exploratory data analysis, data visualization helps researchers formulate new theories and hypotheses ${ }^{16}$. In model assessment, data visualization supports the detection of model misfit and guides

the development of appropriate statistical models (e.g., ${ }^{17-21}$ ). Finally, once the analysis is complete, visualization of data and model fit is arguably the most effective way to communicate the main findings to a scientific audience ${ }^{22}$.

For an example of how data visualization facilitated the development of a new hypothesis, consider the famous "map of the distribution of deaths from cholera" created by London anaesthetist Dr. John Snow during the cholera outbreak in Soho, London in September 1854. In order to trace the source of the outbreak, Dr. Snow created a dot map that displayed the homes of the deceased as well as the water pumps in the neighborhood (Figure 1). The scatter of the data showed that the deaths clustered around a particular water pump in Broad Street, suggesting that the disease was waterborne instead of airborne ${ }^{23}$. Upon Dr. Snow's request, the pump was disabled by removing its handle, which immediately ended the neighbourhood epidemic. It was discovered later that the well belonging to the pump was contaminated with sewage, which caused the outbreak in the neighborhood.

For an example of how data visualization can reveal model misspecification, consider 


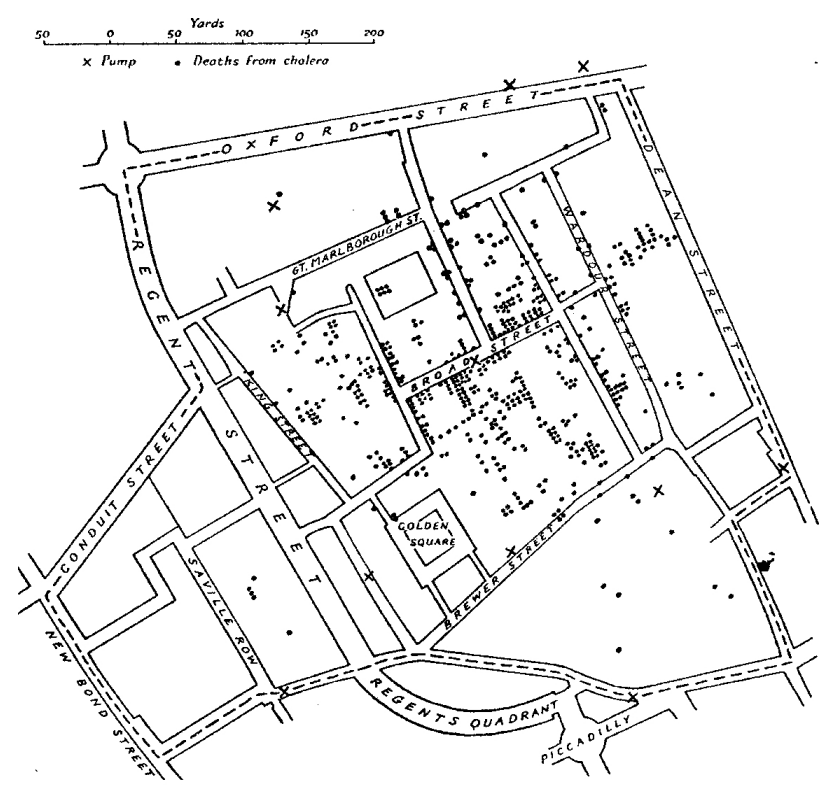

Figure 1: Recreation of Dr. Snow's map of the distribution of deaths from cholera. Points represent homes of the deceased and crosses represent water pumps. The contaminated water pump that triggered the cholera epidemic is located on Broad Street. Reprinted with permission from Pioneer maps of health and disease in England (p. 174), by E. W. Gilbert, 1958, The Royal Geographical Society (with the Institute of British Geographers).

Anscombe's quartet ${ }^{24}$ shown in Figure 2. The four scatter plots all have identical summary statistics (i.e., means, standard deviations, and Pearson correlation coefficient). By visually inspecting the panels, it becomes obvious that the bivariate relation is fundamentally different for each panel (see also ${ }^{25}$ ). 


\subsection{Current Status}

Since William Playfair (1759-1823) invented the first statistical graphs -such as line graphs and bar charts ${ }^{26}$ - data visualization has become an essential part of science. Today, graphs are part of most statistical software packages and have become an indispensable tool to perform certain analyses (e.g., principal component analysis, or prior and posterior predictive checks), or for handling big data sets (e.g., through cluster analysis ${ }^{27}$ ). Technology now allows us to go beyond static visualizations and display the dynamic aspects of the data, for instance, by using the software packages R Shiny ${ }^{28}$ or iNZight ${ }^{29}$.

\subsection{Limitations}

Despite the obvious benefits, data visualization also offers the opportunity to mislead, for instance, when displaying spurious patterns by either expanding the scale to minimize variation, or by minimizing the scale to accentuate differences (e.g., ${ }^{30-32}$ ).

Furthermore, the informativeness of a graph often depends on the design capabilities of the researcher and how much thought they put into what information should be communicated. Scientists without programming experience often find themselves constrained by the options offered in standard graphics software. However, the example of Anscombe's quartet shows that even the simplest plots can be highly informative.

\subsection{Guidelines}

There are no uniform guidelines as to when and which graphical representations should be used. There is, however, a fundamental principle of good statistical graphics due to

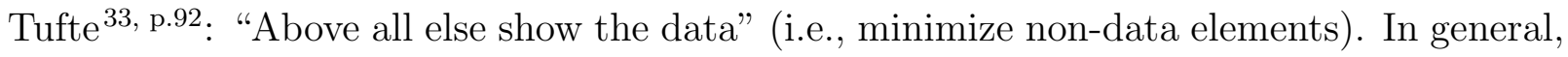
scientists should aim to create a graph that is as clean, informative, and as complete as 

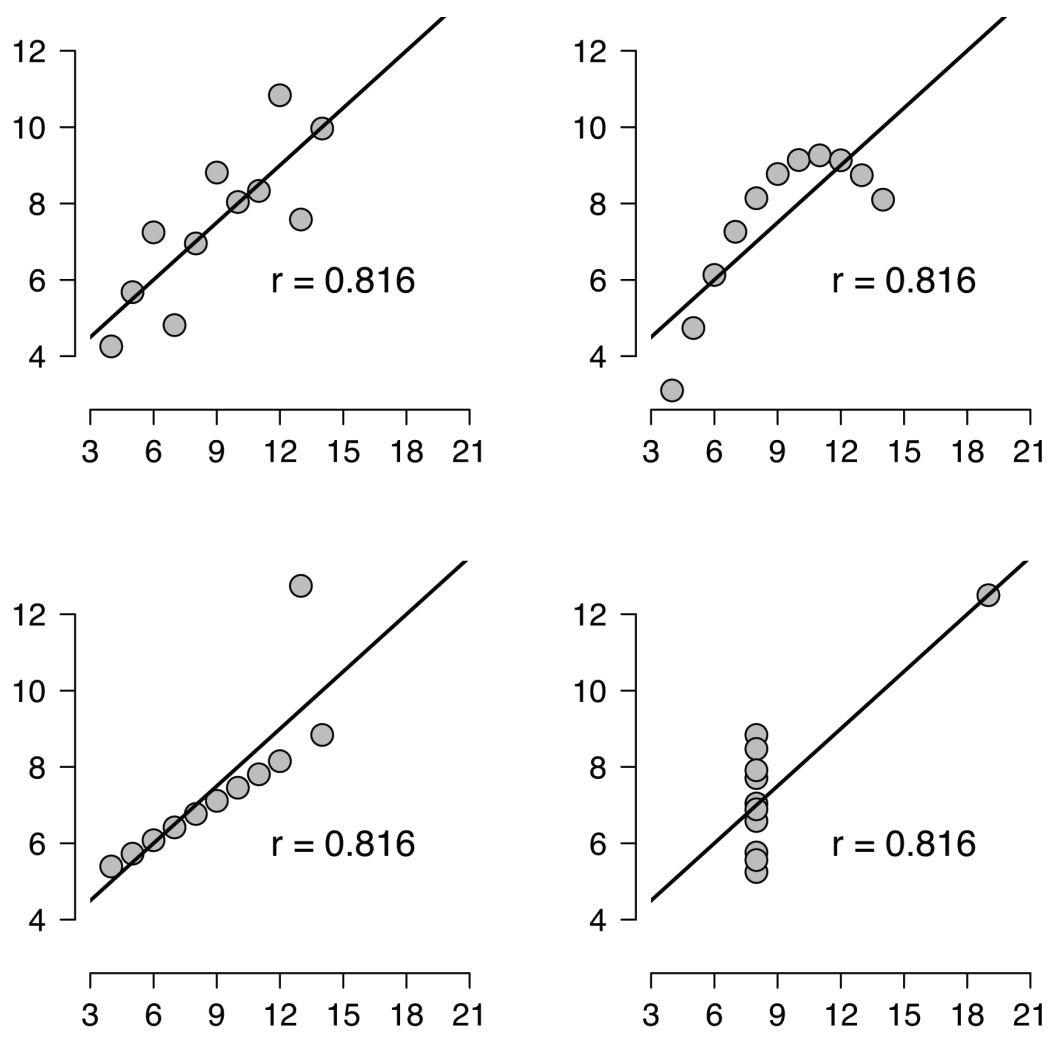

Figure 2: Anscombe's quartet emphasizes the importance of data visualization to detect model misspecification. Although the four data sets are equivalent in terms of their summary statistics, the Pearson correlation is only appropriate for the data set in the upper left panel. 
possible. These characteristics are also emphasized in the ASA Ethical Guidelines ${ }^{34}$. The guidelines mention that to ensure the integrity of data and methods, the ethical statistician "[i]n publications and reports, conveys the findings in ways that are both honest and meaningful to the user/reader. This includes tables, models, and graphics" (p. 3).

Beyond that, guidelines depend on the individual aspects of the data (e.g., complexity of the data and experimental design) and context (cf. ${ }^{35}$ ); here we refer the interested reader to the numerous manuals describing good practices in graphical representation of statistical information (e.g., ${ }^{33 ; 36-41}$ ).

\section{QUANTIFYING INFERENTIAL UNCERTAINTY}

\subsection{Description}

By reporting the precision with which model parameters are estimated, the analyst communicates the inevitable uncertainty that accompanies any inference from a finite sample.

\subsection{Benefits and Example}

Only by assessing and reporting inferential uncertainty is it possible to make any claim about the degree to which results from the sample generalize to the population. For example, Strack et al. ${ }^{42}$ studied whether participants rate cartoons to be funnier when they hold a pen with their teeth (which induces a smile) instead of holding it with their lips (which induces a pout). On a 10-point Likert scale, the authors observed a raw effect size of 0.82 units. For the interpretation of this result it is essential to know the associated inferential uncertainty. In this case, the $95 \%$ confidence interval ranges from -0.05 to 1.69 , indicating that the data are not inconsistent with a large range of effect size estimates (including 
effect sizes that are negligible or negative).

\subsection{Current Status}

In virtually all statistics courses, students are taught to provide not only the summary of statistical tests (such as $F$-, $t$-, $p$-values and associated degrees of freedom), but also parameter point-estimates (e.g., regression weights, effect sizes) and their associated uncertainty (e.g., standard error, posterior distribution, confidence intervals, credible intervals). Nevertheless, there exists a gap between what is taught and what is practiced. Studies of

published articles in physiology ${ }^{21}$, the social sciences ${ }^{43}$, and medicine ${ }^{44 ; 45}$ revealed that error bars, standard errors, or confidence intervals were not always presented. Also, popular metrics such as Cronbach's alpha (a measure of test score reliability) are virtually never presented with a measure of inferential uncertainty.

\subsection{Limitations}

We agree with Jeffreys's comment in the epigraph that there are no acceptable excuses for omitting a measure of inferential uncertainty in any report.

Although not a limitation per se, it should be noted that inferential uncertainty always needs to be quantified relative to the inferential goal: does a researcher want to generalize across people, stimuli, time points, or another dimension? The proper way of computing standard errors depends on the researcher's purpose.

\subsection{Guidelines}

Various guidelines strongly recommend that effect size estimates are accompanied by measures of uncertainty in the form of standard errors or confidence intervals. For instance, 
the publication manual of the American Psychological Association (6th ed.) states: "When point estimates (e.g., sample means or regression coefficients) are provided, always include an associated measure of variability (precision), with an indication of the specific measure used (e.g., the standard error)," (p. 34). Also, the International Committee of Medical Journal Editors ${ }^{46}$ explicitly recommend to "[w] hen possible, quantify findings and present them with appropriate indicators of measurement error or uncertainty (such as confidence intervals)" (p. 17).

\section{Seven Mertonian Statistical Procedures}

This box outlines how each of the seven procedures discussed in the main manuscript fullfill the Mertonian norms. An overview is given in Table 1.

\begin{tabular}{lcccc}
\hline & Communalism & Universalism & Disinterestedness & Organized \\
& & & Skepticism \\
\hline 1. Visualizing Data & $\checkmark$ & $\checkmark$ & $\checkmark$ \\
2. Quantifying Inferential Uncertainty & $\checkmark$ & $\checkmark$ & $\checkmark$ \\
3. Assessing Data Preprocessing Choices & $\checkmark$ & $\checkmark$ & $\checkmark$ \\
4. Reporting Multiple Models & $\checkmark$ & $\checkmark$ & $\checkmark$ \\
5. Involving Multiple Analysts & & $\checkmark$ & $\checkmark$ \\
6. Interpreting Results Modestly & & $\checkmark$ & $\checkmark$ \\
7. Sharing Data and Code & $\checkmark$ & $\checkmark$ & $\checkmark$
\end{tabular}




\section{Visualizing Data}

Well-designed visualizations show at a glance the key aspects of the data. Moreover, by giving the reader a more complete picture of the data and related statistics, visualizations can either support or weaken a conclusion drawn by the researcher, or help the reader find alternative ways of interpreting the results and analyzing the data.

\section{Quantifying Inferential Uncertainty}

Acknowledging inferential uncertainty (e.g., by presenting standard errors or confidence intervals) contributes to open communication. In addition, quantifying inferential uncertainty signals that researchers are openly acknowledging the extent to which their measurements are imprecise, especially when sample size is small. Finally, explicitly acknowledging inferential uncertainty may prompt readers to question how well the results from the sample generalize to the population.

\section{Assessing Data Preprocessing Choices}

When researchers share the results from only a single data pre-processing pipeline, they may unintentionally hide important information. If a result proves sensitive to particular pre-processing choices, this warrants skepticism and may initiate a debate on the importance and plausibility of relevant data pre-processing choices (cf. ${ }^{47,}$ p. ${ }^{308}$ ). 


\section{Reporting Multiple Models}

Similar to the previous section, reporting results from only a single model may unintentionally hide important information.

\section{Involving Multiple Analysts}

The multiple-analysts approach can reveal whether different (teams of) analysts reach converging or diverging conclusions from the same data set. By including other analysts with different backgrounds and interests, the potential impact of self-interest of any single analyst is counteracted. The multiple-analysts approach also stimulates skepticism by bringing to light alternative statistical perspectives on the data.

\section{Interpreting Results Modestly}

Disinterested analysts arguably have little need to exaggerate claims, impress reviewers, and downplay signs of model misfit. Analysts who facilitate organized skepticism do not attempt to suppress doubt - they are not defensive, and they do not wish to protect their work against good-faith scrutiny from their peers.

\section{Sharing Data and Code}

All secrecy about data is a limitation to knowledge accumulation and violates the ethos of science. All interested researchers should have access to relevant, properly anonymized data. Importantly, sharing data allows skeptical eyes to scrutinize the results, promoting quality control. 


\section{ASSESSING DATA PREPROCESSING CHOICES}

\subsection{Description}

By assessing the impact of plausible alternative data pre-processing choices (i.e., examining the "data multiverse" ${ }^{48}$ ), the analyst determines the extent to which the finding under scrutiny is either fragile or sturdy.

\subsection{Benefits and Example}

A "data multiverse" analysis reveals the fragility or sturdiness of the finding under plausible alternative data pre-processing choices. This prevents researchers from falling prey to hindsight bias and motivated reasoning, which may lead them to unwittingly report only the pre-processing pipeline that yields the most compelling result (e.g. ${ }^{49 ; 50}$ ). But even a completely unbiased analysis will benefit from a "data multiverse" analysis, as it reveals uncertainty that would otherwise remain hidden.

For example, Steegen et al. ${ }^{48}$ reexamined the results of Durante et al. ${ }^{51}$, who reported an interaction between relationship status (i.e., single or not) and menstrual cycle (i.e., fertile or not) on reported religiosity. After applying a series of 180 different data preprocessing procedures (e.g., five different ways to split women into high versus low fertility), the multiverse reanalysis showed that the resulting $180 p$-values were distributed uniformly between 0 and 1, indicating that the reported interaction is highly fragile.

\subsection{Current Status}

The idea of assessing sensitivity to data-preprocessing choices dates back at least to De Groot $^{49, \text { p. } 190}$ and Leamer ${ }^{47, \text { p. } 308}$ and was revived by Simmons et al. ${ }^{50}$ and by Steegen 
et al. ${ }^{48}$. In the field of functional magnetic resonance imaging, both Carp ${ }^{52}$ and Poldrack et al. ${ }^{53}$ emphasized the hidden influence of different plausible pre-processing pipelines. In psychology, recent applications are Bastiaansen et al. ${ }^{3}$ and Wessel et al. ${ }^{54}$. Nevertheless, the overwhelming majority of empirical articles does not report the results of a data multiverse analysis.

\subsection{Limitations}

A pragmatic limitation of the data multiverse lies in the extra work that it entails. Another limitation can be found in ambiguities surrounding the definition of the data multiverse. The analyst has to determine what constitutes a sufficiently representative set of preprocessing choices and whether all pre-processing choices are equally plausible, such that they should be given equal weight in the multiverse analysis. A final limitation is that it is not always clear how to interpret the results of a data multiverse analysis. Interpretation can be facilitated with certain graphical formats that cluster related pipelines (e.g., specification curves; $\left.{ }^{55}\right)$.

\subsection{Guidelines}

Some specific guidelines on assessing data pre-processing choices are offered by Simmons et al. ${ }^{50}$, see Requirements for Authors, numbers 5 and 6 , but it is difficult to provide general guidelines as " $(\cdots)$ a multiverse analysis is highly context-specific and inherently subjective. Listing the alternative options for data construction requires judgment about which options can be considered reasonable and will typically depend on the experimental design, the research question, and the researchers performing the research" 48, p. 709 . More general guidelines that relate exclusively to the reporting of pre-processing choices are given in the 
ASA Ethical Guidelines ${ }^{34}$. These mention that to insure the integrity of data and methods, the ethical statistician "[w] hen reporting on the validity of data used, acknowledges data editing procedures, including any imputation and missing data mechanisms" (p. 2).

\section{REPORTING MULTIPLE MODELS}

\subsection{Description}

By assessing the impact of plausible alternative statistical models (i.e., examining the "model multiverse"), the analyst gauges the extent to which a statistical conclusion is either fragile or sturdy.

\subsection{Benefits and Example}

Similar to the "data multiverse" analysis discussion in the previous section, a model multiverse analysis examines the fragility or sturdiness of the finding under plausible alternative statistical modeling choices. Modeling choices comprise differences in estimators and fitting regimes, but also in model specification and variable selection. Reporting the outcomes of multiple plausible models reveals uncertainty that would remain hidden if only a single model were entertained. In addition, this practice protects analysts against hindsight bias and motivated reasoning, which may unwittingly lead them to select the single model that produces the most flattering conclusion. For example, Patel et al. ${ }^{56}$ quantified the variability of results under different model specifications. They considered 13 clinical, environmental, and physiological variables as potential covariates for the association of 417 self-reported, clinical, and molecular phenotypes with all-cause mortality. Consequently, they computed $p$-values for $2^{13}=8,192$ models and examined the instability of the infer- 
ence, which they call the "vibration of effects".

\subsection{Current Status}

Although the idea of the model multiverse dates back at least to De Groot (1956/2014) and Leamer $^{47}$, most empirical researchers still base their conclusion on only a single analysis (but see ${ }^{57 ; 58}$ ).

\subsection{Limitations}

As was the case for the construction of the data multiverse, a pragmatic limitation of the model multiverse lies in the extra work that it entails — for the analyst as well as the reader. Recent work suggests that the number of plausible models can be very large (i.e., ${ }^{4 ; 7}$ ). Also, multiverses vary in their informativeness, and readers need to assess themselves whether a multiverse features notably distinct models or just runs the essentially same model multiple times. Model spaces can be overwhelming; any single analyst will naturally be drawn towards the subset of models that they are familiar with (or, unwittingly, the subset of models that yields the result that is most flattering or most in line with prior expectations). In addition, Del Giudice et al. (in press, p. 5) argue that "By inflating the size of the analysis space, the combinatorial explosion of unjustified specifications may, ironically, exaggerate the perceived exhaustiveness and authoritativeness of the multiverse while greatly reducing the informative fraction of the multiverse. At the same time, the size of the specification space can make it harder to inspect the results for potentially relevant findings. If unchecked, multiverse-style analyses can generate analytic "black holes": Massive analyses that swallow true effects of interest but, due to their perceived exhaustiveness and sheer size, trap whatever information is present in impenetrable displays and summaries." 


\subsection{Guidelines}

Because the construction of the model multiverse depends on the knowledge and expertise of the analyst, it is challenging to provide general guidelines. For relatively simple regression models, however, clear guidelines do exist (e.g., ${ }^{5660}$ ). Furthermore, Simonsohn et al. ${ }^{55}$ suggested a specification curve analysis, and Dragicevic et al. ${ }^{61}$ suggest interactive ways of presenting the results. The ASA Ethical Guidelines ${ }^{34}$ mention that to meet the responsibilities towards funders and clients, the ethical statistician "[t]o the extent possible, presents a client or employer with choices among valid alternative statistical approaches that may vary in scope, cost, or precision" (p. 3). The ASA, however, does not mention that researchers share the same responsibility towards their scientific colleagues, although this may be implicit.

One general recommendation for constructing a comprehensive model multiverse is to collaborate with statisticians who have complementary expertise, bringing us to the next section.

\section{INVOLVING MULTIPLE ANALYSTS}

\subsection{Description}

By having multiple analysts independently analyze the same data set, the researcher can decrease the impact of analyst-specific choices regarding data pre-processing and statistical modeling. 


\subsection{Benefits and Example}

The multiple-analysts approach reveals the uncertainty that is due to the subjective choices of a single analyst and promotes the application of a wider range of statistical techniques. When the conclusions of the analysts converge, this bolsters one's confidence that the finding is robust; when the conclusions diverge, this undercuts that confidence and stimulates a closer look at the statistical reasons for the lack of consensus.

The multiple-analysts approach was used, for example, in a study by Silberzahn et al. ${ }^{7}$ where 29 teams of analysts examined, using the same dataset, whether the skin tone of soccer players influences their probability of getting a red card. While most of the analysis teams reported that players with a darker skin tone have a higher probability of getting a red card, some of the teams reported null results. The analysis approach used by the teams differed widely, both with respect to data pre-processing and statistical modeling (e.g., included covariates, link functions, assumption of hierarchical structure).

\subsection{Current Status}

A precursor to the multiple-analysts approach concerns the 1857 "Cuneiform competition", where four scholars independently translated a previously unseen ancient Assyrian inscription (Rawlinson et al., 1857). The overlap between their translations - sent to the Royal Asian Society in sealed envelopes, and simultaneously opened and inspected by a separate committee of examiners- was striking and put to rest any doubts concerning the method used to decipher such inscriptions. The multiple-analysts approach never caught on in practice, although recent examples exist in psychology and neuroscience ${ }^{3-5 ; 7 ; 62 ; 63}$. 


\subsection{Limitations}

As was the case for the construction of the data multiverse and the model multiverse, a pragmatic limitation of the multiple analyst approach lies in the extra work that it entails, specifically with respect to (1) finding knowledgeable analysts who are interested in participating; (2) documenting the data set, describing the research question, and identifying the target of statistical inference; (3) collating the initial responses from each team, and potentially coordinating a review and feedback round. While differences in opinion should be respected, there need to be ways to filter out analysis approaches that involve clear mistakes. An additional limitation concerns possible homogeneity of the analysts. For instance, all analysts involved could be rigidly educated in the same school of thought, share cultural or social biases, or just make the same mistake. In such a case, the results may create an inflated sense of certainty in the conclusion that was reached. This potential limitations can be mitigated by selecting a diverse group of analysts and incorporating feedback and revision options in the process ${ }^{7}$, a round-table discussion ${ }^{5}$ or, more systematically, a Delphi approach ${ }^{64}$.

\subsection{Guidelines}

There are no explicit guidelines concerning the multiple-analysts approach. We propose that the optimal number of analysts to include depends on factors such as the complexity of the data, the importance of the research question (e.g., a clinical trial on the effectiveness of a new drug against COVID-19 warrants a relatively large number of analysts), and the

probability that the analysts could reasonably reach a different conclusion (e.g., there may be multiple ways to interpret the research question, and there may be multiple dependent variables and predictor variables that could or could not be relevant). 
When analysts are selected, care should be taken to ensure heterogeneity, diversity, and balance. Specifically, one should be mindful of the potential biasing effects of specific background knowledge, culture, education, and career stage of the analyst.

The ASA Guidelines emphasize the legitimacy and value in alternative analytic approaches, stating that "[t]he practice of statistics requires consideration of the entire range of possible explanations for observed phenomena, and distinct observers $(\cdots)$ can arrive at different and potentially diverging judgments about the plausibility of different explanations" (p. 5).

\section{INTERPRETING RESULTS MODESTLY}

\section{$7.1 \quad$ Description}

By modestly interpreting the results, the analyst explicitly acknowledges any remaining doubts concerning the importance, replicability, and generalizability of the scientific claims at hand.

\subsection{Benefits and Example}

Modestly presented scientific claims enable the reader to evaluate the outcomes for what they usually are: not final, but tentative results pointing in a certain direction, with considerable uncertainty surrounding their generalizability and scope. Overselling results might lead to the misallocation of public resources towards approaches that are in fact not properly validated and not ready for application in practice. Also, researchers themselves risk losing long-term credibility for short-term gains of greater attention and higher citation counts. Moreover, after having publicly committed to a bold claim, it becomes difficult 
to admit that one's initial assessment was wrong; in other words, overconfidence is not conducive to scientific learning.

Scientists of true modesty remain doubtful even at moments of great success. For example, when James Chadwick found experimental proof of neutrons, the discovery that earned him the Nobel prize, he communicated it modestly under the title "Possible Existence of Neutron" 65 .

\subsection{Current Status}

Tukey ${ }^{66}$ already remarked that "Laying aside unethical practices, one of the most dangerous $[(\cdots)$ practices of data analysis $(\cdots)]$ is the use of formal data-analytical procedures for sanctification, for the preservation of conclusions from all criticism, for the granting of an imprimatur." (p. 13). Almost 60 years later, an editorial in Nature Human Behaviour warns its readers about "conclusive narratives that leave no room for ambiguity or for conflicting or inconclusive results" 67, p. 1 . Similarly, Simons et al. ${ }^{68}$ suggested adding a mandatory Constraints on Generality statement in the discussion section of all primary research articles in the field of psychology to prevent authors from making wildly exaggerated claims of generality. This suggests that scientific modesty is rarer than we would expect if Mertonian norms were widely adopted. There are some clear indications of a lack of modesty. First of all, the frequency of stronger language (words like "amazing", "groundbreaking", "unprecedented") seemed to have increased in the last few decades ${ }^{69}$. Secondly, dichotomization of findings (i.e., ignoring the uncertainty inherent to statistical inference) is common practice (e.g., ${ }^{43}$; also see paragraph 4.3). Thirdly, textbooks (which are typically a reflection of current practice) on how to write papers often explicitly encourage authors to overclaim (e.g., $\left.{ }^{70 ; 71}\right)$. 


\subsection{Limitations}

Publications and grants are important for scientific survival. Coupled with the fact that journals and funders often prefer groundbreaking and unequivocal outcomes, it may be detrimental to one's success to modestly interpret the results. The encouragement of this Mertonian practice may require change at an institutional level, although some have argued that scientists should not hide behind the system when defending their behavior ${ }^{72}$.

\subsection{Guidelines}

There are several ways we can contribute to increasing intellectual modesty. First of all, we could encourage intellectual modesty in others' work when we act as reviewers of papers and grant proposals ${ }^{73}$. Since a reviewer's career is independent of how they evaluate a paper, they can make a positive review conditional on a more modest presentation of outcomes. Hoekstra and Vazire ${ }^{73}$ present a list of suggestions for increasing modesty in the traditional sections of an empirical article, which can be used by authors as well. One example (p. 16)

includes "Titles should not state or imply stronger claims than are justified (e.g., causal claims without strong evidence)".

Also, the ASA Guidelines state: "[t]he ethical statistician is candid about any known or suspected limitations, defects, or biases in the data that may affect the integrity or reliability of the statistical analysis" (p. 2). 


\section{SHARING DATA AND CODE}

\subsection{Description}

By sharing data and analysis code, researchers provide the basis for their scientific claims. Ideally, data and code should be shared publicly, freely, and in a manner that facilitates reuse.

\subsection{Benefits and Example}

Since there are many different ways of processing and analyzing data ${ }^{7 ; 48}$, sharing code promotes reproducibility and encourages sensitivity analyses. Sharing data and code also allows other researchers to establish the validity of the original analyses, it can facilitate collaboration, but it can also serve as protection against data loss. When publishing his theory on "general intelligence", Spearman ${ }^{74}$ shared his data as an appendix to the article. A century later, this act of foresight enabled scientists to use this data set for both research and education. Because Spearman made his data publicly available, other researchers could establish the reproducibility and generalizability of the findings.

\subsection{Current Status}

Data sharing has never been easier. Public repositories offer free storage space for research materials, data (e.g., the Open Science Framework), and code (e.g., Github). While data sharing is not yet a general practice in most scientific fields, several recent initiatives (e.g., Open Data/Code/Materials badges, ${ }^{75}$ ), standards (TOP Guidelines, ${ }^{76}$ ), journals (e.g., Sci-

entific Data) and checklists (e.g., Transparency Checklist, ${ }^{77}$ ) are helping to promote this research practice. When sharing raw data is unfeasible, researchers can make aggregated 
data summaries available, for example, the data used to generate certain plots or covariance matrices of involved variables.

\subsection{Limitations}

Restrictions imposed by funders, ethics review boards in universities and other institutions, collaborators, and legal contracts may limit the extent to which data can be publicly shared. There may also be practical considerations (e.g., sharing big data), data use agreements, privacy rights, and institutional policies that can curtail sharing intentions. What remains central is to inform the readers about the accessibility of the data of the analysis. It should be noted that these limitations should not apply to the analysis code as long as code is solely reflective of the researcher's analysis actions and is free of any data privacy issues.

\subsection{Guidelines}

An important principle of sharing data is that they should be Findable, Accessible, Interoperable, and Reusable (FAIR, $\left.{ }^{78}\right)$. Several guides are available discussing the practical (e.g., ${ }^{79}$ ) and ethical (e.g., ${ }^{80}$ ) aspects of data sharing. Researchers should follow the data sharing procedures and requirements of their fields (e.g., ${ }^{81 ; 82}$ ) and indicate the accessibility of the data in the research report ${ }^{76 ; 83}$. The ASA Ethical Guidelines ${ }^{34}$ for Statistical Practice state that the ethical statistician "[p]romotes sharing of data and methods as much as possible", and "[m]akes documentation suitable for replicate analyses, metadata studies, and other research by qualified investigators." (p. 5). 


\section{CONCLUDING COMMENTS}

If the statistical literature is any guide, one may conclude that statisticians rarely agree with one another. For instance, the 2019 special issue in The American Statistician featured 43 articles on $p$-values, and in their editorial Wasserstein et al. ${ }^{13}$ stated that "the voices in the 43 papers in this issue do not sing as one". However, despite the continuing disagreements about the foundations of statistical inference, we believe there is nevertheless much common ground among statisticians, specifically with respect to the ethical aspects of their profession. To explore this ethical dimension more systematically, we started by considering the Mertonian norms that characterize the ethos of science and outlined a non-

exhaustive list of seven concrete, teachable, and implementable practices that we believe need wider propagation.

In essence, these practices are about promoting transparency and the open acknowledgement of uncertainty. With agreement on such practices explicitly acknowledged, we believe that commonly discussed contentious issues (e.g., $p$-values) may become less crucial. Indeed, in a letter to his frequentist nemesis Sir Ronald Fisher, the arch-Bayesian Sir Harold Jeffreys wrote "Your letter confirms my previous impression that it would only be once in a blue moon that we would disagree about the inference to be drawn in any particular case, and that in the exceptional cases we would both be a bit doubtful" ${ }^{84, \text { p. }} 162$.

We hope that the proposed statistical practices will improve the quality of data analysis across the board, especially in applied disciplines that are perhaps unfamiliar with the ethical aspects of statistics, aspects that a statistician may take for granted. Also, instead of counting on them to be absorbed through osmosis, we believe it is important to include these ethical considerations -and their statistical consequences- explicitly in the statistics curricula. Statistical techniques other than those discussed here may also further 
the Mertonian ideals. We hope that this contribution provides the impetus for a deeper exploration of how data analysis in applied fields can become more transparent, more informative, and more open about the uncertainties that inevitably arise in any statistical data analysis problem.

\section{AUTHOR CONTRIBUTIONS}

Conceptualization: E.-J.W., A. Sarafoglou, and B.A.

Project Administration: B.A.

Writing - Original Draft Preparation: E.-J.W., A. Sarafoglou, C.A., J.A., Š.B., N.v.D., R.H., D.M., D.v.R., A. Sluga, J.N.T., and B.A.

Writing - Review \& Editing: E.-J.W., A. Sarafoglou, S.A., C.A., J.A., Š.B., N.v.D., R.H., D.M., D.v.R., A. Sluga, F.S., J.N.T., and B.A.

\section{ACKNOWLEDGEMENTS}

We are grateful to Nicole Lazar for her comments on a draft version. We also thank everyone who was involved in drafting the initial list of statistical procedures during the hackathon that took place at the 2019 meeting of the Society for the Improvement of Psychological Science in Rotterdam, The Netherlands. This work was supported in part by a European Research Council (ERC) grant to E.-J.W. (283876), a Netherlands Organisation for Scientific Research (NWO) grant to A. Sarafoglou (406-17-568), as well as a Dutch scientific organization Vidi grant from the NWO to D.v.R. (016.Vidi.188.001). 


\section{COMPETING INTERESTS}

The authors declare no competing interests. 


\section{References}

[1] I. J. Good. 46656 varieties of Bayesians. The American Statistician, 25:62-63, 1971.

[2] B. Aczel, R. Hoekstra, A. Gelman, E.-J. Wagenmakers, I. G. Klugkist, J. N. Rouder, J. Vandekerckhove, M. D. Lee, R. D. Morey, W. Vanpaemel, Z. Dienes, and D. van Ravenzwaaij. Discussion points for Bayesian inference. Nature Human Behaviour, 4: 561-566, 2020. URL https://doi.org/10.1038/s41562-019-0807-z.

[3] Jojanneke A Bastiaansen, Yoram K Kunkels, Frank J Blaauw, Steven M Boker, Eva Ceulemans, Meng Chen, Sy-Miin Chow, Peter de Jonge, Ando C Emerencia, Sacha Epskamp, et al. Time to get personal? The impact of researchers choices on the selection of treatment targets using the experience sampling methodology. Journal of Psychosomatic Research, 137:110211, 2020.

[4] R. Botvinik-Nezer, F. Holzmeister, C. F. Camerer, A. Dreber, J. Huber, M. Johannesson, M. Kirchler, R. Iwanir, J. A. Mumford, A. Adcock, P. Avesani, B. Baczkowski, A. Bajracharya, L. Bakst, S. Ball, M. Barilari, N. Bault, D. Beaton, J. Beitner, R. Benoit, R. Berkers, J. Bhanji, B. Biswal, S. Bobadilla-Suarez, T. Bortolini, K. Bottenhorn, A. Bowring, S. Braem, H. Brooks, E. Brudner, C. Calderon, J. Camilleri, J. Castrellon, L. Cecchetti, E. Cieslik, Z. Cole, O. Collignon, R. Cox, W. Cunningham, S. Czoschke, K. Dadi, C. Davis, A. De Luca, M. Delgado, L. Demetriou, J. Dennison, X. Di, E. Dickie, E. Dobryakova, C. Donnat, J. Dukart, N. W. Duncan, J. Durnez, A. Eed, S. Eickhoff, A. Erhart, L. Fontanesi, G. M. Fricke, A. Galvan, R. Gau, S. Genon, T. Glatard, E. Glerean, J. Goeman, S. Golowin, C. González-García, K. Gorgolewski, C. Grady, M. Green, J. Guassi Moreira, O. Guest, S. Hakimi, J. P. Hamilton, R. Hancock, G. Handjaras, B. Harry, C. Hawco, P. Herholz, G. Her- 
man, S. Heunis, F. Hoffstaedter, J. Hogeveen, S. Holmes, C.-P. Hu, S. Huettel, M. Hughes, V. Iacovella, A. Iordan, P. Isager, A. I. Isik, A. Jahn, M. Johnson, T. Johnstone, M. Joseph, A. Juliano, J. Kable, M. Kassinopoulos, C. Koba, X.-Z. Kong, T. Koscik, N. E. Kucukboyaci, B. Kuhl, S. Kupek, A. Laird, C. Lamm, R. Langner, N. Lauharatanahirun, H. Lee, S. Lee, A. Leemans, A. Leo, E. Lesage, F. Li, M. Li, P. C. Lim, E. Lintz, S. Liphardt, A. Losecaat Vermeer, B. Love, M. Mack, N. Malpica, T. Marins, C. Maumet, K. McDonald, J. McGuire, H. Melero, A. Méndez Leal, B. Meyer, K. Meyer, P. Mihai, G. Mitsis, J. Moll, D. Nielson, G. Nilsonne, M. Notter, E. Olivetti, A. Onicas, P. Papale, K. Patil, J. E. Peelle, A. Pérez, D. Pischedda, J.-B. Poline, Y. Prystauka, S. Ray, P. Reuter-Lorenz, R. Reynolds, E. Ricciardi, J. Rieck, A. Rodriguez-Thompson, A. Romyn, T. Salo, G. Samanez-Larkin, E. SanzMorales, M. Schlichting, D. Schultz, Q. Shen, M. Sheridan, F. Shiguang, J. Silvers, K. Skagerlund, A. Smith, D. Smith, P. Sokol-Hessner, S. Steinkamp, S. Tashjian, B. Thirion, J. Thorp, G. Tinghög, L. Tisdall, S. Tompson, C. Toro-Serey, J. Torre, L. Tozzi, V. Truong, L. Turella, A. E. van’t Veer, T. Verguts, J. Vettel, S. Vijayarajah, K. Vo, M. Wall, W. D. Weeda, S. Weis, D. White, D. Wisniewski, A. XifraPorxas, E. Yearling, S. Yoon, R. Yuan, K. Yuen, L. Zhang, X. Zhang, J. Zosky, T. E. Nichols, R. A. Poldrack, and T. Schonberg. Variability in the analysis of a single neuroimaging dataset by many teams. Nature, 582:84-88, 2020. URL https://doi.org/10.1038/s41586-020-2314-9.

[5] N. van Dongen, J. B. van Doorn, Q. F. Gronau, D. van Ravenzwaaij, R. Hoekstra, M. N. Haucke, D. Lakens, C. Hennig, R. D. Morey, S. Homer, A. Gelman, J. Sprenger, and E.-J. Wagenmakers. Multiple perspectives on inference for two simple statistical scenarios. The American Statistician, 73:328-339, 2019. 
[6] Matthew J. Salganik, Ian Lundberg, Alexander T. Kindel, Caitlin E. Ahearn, Khaled Al-Ghoneim, Abdullah Almaatouq, Drew M. Altschul, Jennie E. Brand, Nicole Bohme Carnegie, Ryan James Compton, Debanjan Datta, Thomas Davidson, Anna Filippova, Connor Gilroy, Brian J. Goode, Eaman Jahani, Ridhi Kashyap, Antje Kirchner, Stephen McKay, Allison C. Morgan, Alex Pentland, Kivan Polimis, Louis Raes, Daniel E. Rigobon, Claudia V. Roberts, Diana M. Stanescu, Yoshihiko Suhara, Adaner Usmani, Erik H. Wang, Muna Adem, Abdulla Alhajri, Bedoor AlShebli, Redwane Amin, Ryan B. Amos, Lisa P. Argyle, Livia Baer-Bositis, Moritz Büchi, Bo-Ryehn Chung, William Eggert, Gregory Faletto, Zhilin Fan, Jeremy Freese, Tejomay Gadgil, Josh Gagné, Yue Gao, Andrew Halpern-Manners, Sonia P. Hashim, Sonia Hausen, Guanhua He, Kimberly Higuera, Bernie Hogan, Ilana M. Horwitz, Lisa M. Hummel, Naman Jain, Kun Jin, David Jurgens, Patrick Kaminski, Areg Karapetyan, E. H. Kim, Ben Leizman, Naijia Liu, Malte Möser, Andrew E. Mack, Mayank Mahajan, Noah Mandell, Helge Marahrens, Diana Mercado-Garcia, Viola Mocz, Katariina Mueller-Gastell, Ahmed Musse, Qiankun Niu, William Nowak, Hamidreza Omidvar, Andrew Or, Karen Ouyang, Katy M. Pinto, Ethan Porter, Kristin E. Porter, Crystal Qian, Tamkinat Rauf, Anahit Sargsyan, Thomas Schaffner, Landon Schnabel, Bryan Schonfeld, Ben Sender, Jonathan D. Tang, Emma Tsurkov, Austin van Loon, Onur Varol, Xiafei Wang, Zhi Wang, Julia Wang, Flora Wang, Samantha Weissman, Kirstie Whitaker, Maria K. Wolters, Wei Lee Woon, James Wu, Catherine Wu, Kengran Yang, Jingwen Yin, Bingyu Zhao, Chenyun Zhu, Jeanne Brooks-Gunn, Barbara E. Engelhardt, Moritz Hardt, Dean Knox, Karen Levy, Arvind Narayanan, Brandon M. Stewart, Duncan J. Watts, and Sara McLanahan. Measuring the predictability of life outcomes with a scientific mass collaboration. Proceedings of the National Academy of Sciences, 117:8398-8403, 2020. doi: 10.1073/pnas.1915006117. 
[7] R. Silberzahn, E. L. Uhlmann, D. P. Martin, P. Anselmi, F. Aust, E. Awtrey, S̆. Bahník, F. Bai, C. Bannard, E. Bonnier, R. Carlsson, F. Cheung, G. Christensen, R. Clay, M. A. Craig, A. Dalla Rosa, L. Dam, M. H. Evans, I. Flores Cervantes, N. Fong, M. Gamez-Djokic, A. Glenz, S. Gordon-McKeon, T. J. Heaton, K. Hederos, M. Heene, A. J. Hofelich Mohr, Högden F., K. Hui, M. Johannesson, J. Kalodimos, E. Kaszubowski, D. M. Kennedy, R. Lei, T. A. Lindsay, S. Liverani, C. R. Madan, D. Molden, E. Molleman, R. D. Morey, L. B. Mulder, B. R. Nijstad, N. G. Pope, B. Pope, J. M. Prenoveau, F. Rink, E. Robusto, H. Roderique, A. Sandberg, E. Schlüter, F. D. Schönbrodt, M. F. Sherman, S. A. Sommer, K. Sotak, S. Spain, Spörlein C., T. Stafford, L. Stefanutti, S. Tauber, J. Ullrich, M. Vianello, E.-J. Wagenmakers, M. Witkowiak, S. Yoon, and B. A. Nosek. Many analysts, one data set: Making transparent how variations in analytic choices affect results. Advances in Methods and Practices in Psychological Science, 1:337-356, 2018.

[8] V. Amrhein, S. Greenland, and B. B. McShane. Retire statistical significance. Nature, 567:305-307, 2019.

[9] D. J. Benjamin, J. O. Berger, M. Johannesson, B. A. Nosek, E.-J. Wagenmakers, R. Berk, K. A. Bollen, B. Brembs, L. Brown, C. Camerer, D. Cesarini, C. D. Chambers, M. Clyde, T. D. Cook, P. De Boeck, Z. Dienes, A. Dreber, K. Easwaran, C. Efferson, E. Fehr, F. Fidler, A. P. Field, M. Forster, E. I. George, R. Gonzalez, S. Goodman, E. Green, D. P. Green, A. Greenwald, J. D. Hadfield, L. V. Hedges, L. Held, T.-H. Ho, H. Hoijtink, J. H. Jones, D. J. Hruschka, K. Imai, G. Imbens, J. P. A. Ioannidis, M. Jeon, M. Kirchler, D. Laibson, J. List, R. Little, A. Lupia, E. Machery, S. E. Maxwell, M. McCarthy, D. Moore, S. L. Morgan, M. Munafò, S. Nakagawa, B. Nyhan, T. H. Parker, L. Pericchi, M. Perugini, J. Rouder, J. Rousseau, V. Savalei, F. D. 
Schönbrodt, T. Sellke, B. Sinclair, D. Tingley, T. Van Zandt, S. Vazire, D. J. Watts, C. Winship, R. L. Wolpert, Y. Xie, C. Young, J. Zinman, and V. E. Johnson. Redefine statistical significance. Nature Human Behaviour, 2:6-10, 2018.

[10] L. L. Harlow, S. A. Mulaik, and J. H. Steiger, editors. What if There Were No Significance Tests? Lawrence Erlbaum, Mahwah (NJ), 1997.

[11] B. B. McShane, D. Gal, A. Gelman, C. Robert, and J. L. Tackett. Abandon statistical significance. The American Statistician, 73:235-245, 2019.

[12] R. L. Wasserstein and N. A. Lazar. The ASA's statement on $p$-values: Context, process, and purpose. The American Statistician, 70:129-133, 2016.

[13] R. L. Wasserstein, A. L. Schirm, and N. A. Lazar. Moving to a world beyond " $p<$ 0.05". The American Statistician, 73:1-19, 2019.

[14] R. K. Merton. The normative structure of science (1942). In R. K. Merton, editor, The Sociology of Science: Theoretical and Empirical Investigations, pages 267-278. University of Chicago Press, Chicago, IL, 1973.

[15] Melissa S Anderson, Brian C Martinson, and Raymond De Vries. Normative dissonance in science: Results from a national survey of us scientists. Journal of Empirical Research on Human Research Ethics, 2:3-14, 2007.

[16] J. W. Tukey. Explanatory Data Analysis. Addison-Wesley, Reading, MA, 1977.

[17] Andrew Gelman. Exploratory data analysis for complex models. Journal of Computational and Graphical Statistics, 13:755-779, 2004. doi: 10.1198/106186004X11435. 
[18] Jonah Gabry, Daniel Simpson, Aki Vehtari, Michael Betancourt, and Andrew Gelman. Visualization in Bayesian workflow. Journal of the Royal Statistical Society: Series A (Statistics in Society), 182:389-402, 2019.

[19] Andrew Heathcote, Scott D Brown, and Eric-Jan Wagenmakers. An introduction to good practices in cognitive modeling. In An Introduction to Model-Based Cognitive Neuroscience, pages 25-48. Springer Verlag, 2015.

[20] J. Kerman, A. Gelman, T. Zheng, and Y. Ding. Visualization in Bayesian data analysis. In C. Chen, W. Härdle, and A. Unwin, editors, Handbook of Data Visualization, pages 709-724. Springer Verlag, Berlin, 2008.

[21] T. L. Weissgerber, N. M. Milic, S. J. Winham, and V. D. Garovic. Beyond bar and line graphs: Time for a new data presentation paradigm. PLoS Biology, 13:e1002128, 2015 .

[22] Kieran Healy and James Moody. Data visualization in sociology. Annual Review of Sociology, 40:105-128, 2014.

[23] Edmund William Gilbert. Pioneer maps of health and disease in England. The Geographical Journal, 124:172-183, 1958.

[24] F. J. Anscombe. Graphs in statistical analysis. The American Statistician, 27:17-21, 1973.

[25] J. Matejka and G Fitzmaurice. Same stats, different graphs: Generating datasets with varied appearance and identical statistics through simulated annealing. Proceedings of the 2017 CHI Conference on Human Factors in Computing Systems, pages 1290-1294, 2017. 
[26] W. Playfair. Commercial and Political Atlas: Representing, by Copper-Plate Charts, the Progress of the Commerce, Revenues, Expenditure, and Debts of England, during the Whole of the Eighteenth Century. Corry, London, 1786. Re-published in Wainer, H. and Spence, I. (eds.), The Commercial and Political Atlas and Statistical Breviary, 2005, Cambridge University Press.

[27] Brian S Everitt, Sabine Landau, Morven Leese, and Daniel Stahl. Cluster Analysis. John Wiley \& Sons, Chichester, 2011.

[28] W Chang, J Cheng, JJ Allaire, Y Xie, and J McPherson. shiny: Web application framework for R [Computer software]. http://CRAN . R-project. org/package=shiny, 2020.

[29] iNZight Team. iNZight (Version 4.0.2.) [Computer software]. https://inzight.nz, 2020.

[30] Alberto Cairo. How Charts Lie: Getting Smarter about Visual Information. WW Norton \& Company, New York, 2019.

[31] Andrew Gelman. Why tables are really much better than graphs. Journal of Computational and Graphical Statistics, 20:3-7, 2011.

[32] Howard Wainer. How to display data badly. The American Statistician, 38:137-147, 1984.

[33] Edward R Tufte. The Visual Display of Quantitative Information. Graphics Press, Cheshire, CT, 1973. 
[34] Committee on Professional Ethics of the American Statistical Association. Ethical guidelines for statistical practice, 2018. URL https://www . amstat.org/ASA/Your-Career/Ethical-Guidelines-for-Statistical-Practice.

[35] L. Diamond and F. J. Lerch. Fading frames: Data presentation and framing effects. Decision Sciences, 23:1050-1071, 1992.

[36] C. Chen, W. Härdle, and A. Unwin, editors. Handbook of Data Visualization. Springer Verlag, Berlin, 2008.

[37] William S Cleveland and Robert McGill. Graphical perception: Theory, experimentation, and application to the development of graphical methods. Journal of the American Statistical Association, 79:531-554, 1984.

[38] Andrew Gelman, Cristian Pasarica, and Rahul Dodhia. Let's practice what we preach: Turning tables into graphs. The American Statistician, 56:121-130, 2002.

[39] Riccardo Mazza. Introduction to information visualization. Springer Science \& Business Media, London, 2009.

[40] Claus O Wilke. Fundamentals of data visualization: A primer on making informative and compelling figures. O'Reilly Media, Sebastopol, CA, 2019.

[41] Leland Wilkinson. The Grammar of Graphics. Springer Science \& Business Media, New York, 1999.

[42] F. Strack, L. L. Martin, and S. Stepper. Inhibiting and facilitating conditions of the human smile: A nonobtrusive test of the facial feedback hypothesis. Journal of Personality and Social Psychology, 54:768-777, 1988. 
[43] Rink Hoekstra, Sue Finch, Henk AL Kiers, and Addie Johnson. Probability as certainty: Dichotomous thinking and the misuse of p-values. Psychonomic Bulletin \& Review, 13:1033-1037, 2006.

[44] Richelle J Cooper, David L Schriger, and Reb JH Close. Graphical literacy: The quality of graphs in a large-circulation journal. Annals of Emergency Medicine, 40: 317-322, 2002.

[45] David L Schriger, Reshmi Sinha, Sara Schroter, Pamela Y Liu, and Douglas G Altman. From submission to publication: A retrospective review of the tables and figures in a cohort of randomized controlled trials submitted to the British Medical Journal. Annals of Emergency Medicine, 48:750-756, 2006.

[46] International Committee of Medical Journal Editors. Recommendations for the conduct, reporting, editing, and publication of scholarly work in medical journals. http://www.icmje.org/icmje-recommendations.pdf, 2019.

[47] Edward E Leamer. Sensitivity analyses would help. The American Economic Review, 75:308-313, 1985.

[48] S. Steegen, F. Tuerlinckx, A. Gelman, and W. Vanpaemel. Increasing transparency through a multiverse analysis. Perspectives on Psychological Science, 11:702-712, 2016.

[49] A. D. De Groot. The meaning of "significance" for different types of research. Translated and annotated by Eric-Jan Wagenmakers, Denny Borsboom, Josine Verhagen, Rogier Kievit, Marjan Bakker, Angelique Cramer, Dora Matzke, Don Mellenbergh, and Han L. J. van der Maas. Acta Psychologica, 148:188-194, 1956/2014. 
[50] J. P. Simmons, L. D. Nelson, and U. Simonsohn. False-positive psychology: Undisclosed flexibility in data collection and analysis allows presenting anything as significant. Psychological Science, 22:1359-1366, 2011.

[51] Kristina M Durante, Ashley Rae, and Vladas Griskevicius. The fluctuating female vote: Politics, religion, and the ovulatory cycle. Psychological Science, 24:1007-1016, 2013.

[52] J. Carp. On the plurality of (methodological) worlds: Estimating the analytic flexibility of fMRI experiments. Frontiers in Neuroscience, 6:1-13, 2012.

[53] R. A. Poldrack, C. I. Baker, J. Durnez, K. J. Gorgolewski, P. M. Matthews, M. R. Munafò, T. E. Nichols, J.-B. Poline, E. Vul, and T. Yarkoni. Scanning the horizon: Towards transparent and reproducible neuroimaging research. Nature Reviews Neuroscience, 18:115-126, 2017.

[54] Ineke Wessel, Casper Albers, Anna Roos Eva Zandstra, and Vera Ellen Heininga. A multiverse analysis of early attempts to replicate memory suppression with the Think/No-think task. Memory, 28:870-887, 2020.

[55] U. Simonsohn, L. D. Nelson, and J. P. Simmons. Specification curve analysis. Nature Human Behaviour, 4:1208-1214, 2020.

[56] Chirag J Patel, Belinda Burford, and John PA Ioannidis. Assessment of vibration of effects due to model specification can demonstrate the instability of observational associations. Journal of Clinical Epidemiology, 68:1046-1058, 2015.

[57] Susan Athey and Guido W Imbens. Machine learning methods that economists should know about. Annual Review of Economics, 11:685-725, 2019. 
[58] Ross Levine and David Renelt. A sensitivity analysis of cross-country growth regressions. The American Economic Review, pages 942-963, 1992.

[59] Marco Del Giudice, Steven W Gangestad, and W Steven. A traveler's guide to the multiverse: Promises, pitfalls, and a framework for the evaluation of analytic decisions. Advances in Methods and Practices in Psychological Science, in press.

[60] Jennifer A Hoeting, David Madigan, Adrian E Raftery, and Chris T Volinsky. Bayesian model averaging: A tutorial. Statistical Science, pages 382-401, 1999.

[61] P. Dragicevic, Y. Jansen, A. Sarma, M. Kay, and F. Chevalier. Increasing the transparency of research papers with explorable multiverse analyses. Proceedings of the 2019 CHI Conference on Human Factors in Computing Systems, pages 1-15, 2019.

[62] U. Boehm, G. E. Hawkins, S. D. Brown, H. van Rijn, and E.-J. Wagenmakers. Of monkeys and men: Impatience in perceptual decision-making. Psychonomic Bulletin E) Review, 23:738-749, 2016.

[63] Gilles Dutilh, Jeffrey Annis, Scott D Brown, Peter Cassey, Nathan J Evans, Raoul PPP Grasman, Guy E Hawkins, Andrew Heathcote, William R Holmes, Angelos-Miltiadis Krypotos, et al. The quality of response time data inference: A blinded, collaborative assessment of the validity of cognitive models. Psychonomic Bulletin $\&$ Review, 26 : 1051-1069, 2019.

[64] Shakila Thangaratinam and Charles WE Redman. The delphi technique. The Obstetrician $\&$ Gynaecologist, 7:120-125, 2005.

[65] James Chadwick. Possible existence of a neutron. Nature, 129:312, 1932. 
[66] J. W. Tukey. The future of data analysis. The Annals of Mathematical Statistics, 33: $1-67,1962$.

[67] NHB Editorial. Tell it like it is. Nature Human Behaviour, 4:1, 2020.

[68] D. J. Simons, Y. Shoda, and D. S. Lindsay. Constraints on generality (cog): A proposed addition to all empirical papers. Perspectives on Psychological Science, 12:1123-1128, 2017.

[69] Christiaan H Vinkers, Joeri K Tijdink, and Willem M Otte. Use of positive and negative words in scientific pubmed abstracts between 1974 and 2014: Retrospective analysis. BMJ, 351:h6467, 2015.

[70] Daryl J Bem. Writing the empirical journal. In M. R. Zanna and J. M. Darley, editors, The compleat academic: A practical guide for the beginning social scientist, pages 171-201. Lawrence Erlbaum Associates, Mahwah, NJ, 1987.

[71] J. van Doorn, D. van den Bergh, F. Dablander, N. van Dongen, K. Derks, N. J. Evans, Q. F. Gronau, J. M. Haaf, Y. Kunisato, A. Ly, M. Marsman, A. Sarafoglou, A. Stefan, and E.-J. Wagenmakers. Strong public claims may not reflect researchers' private convictions. Significance, 2021. URL https://psyarxiv.com/pc4ad.

[72] T Yarkoni. No, it's not the incentives-it's you. https://www.talyarkoni.org/blog/2018/10/02/no-its-not-the-incentives-its-you/, 2018 .

[73] R. Hoekstra and S. Vazire. Intellectual humility is central to science: Some practices to aspire to. PsyArXiv, 2020. 
[74] C Spearman. General intelligence, objectively determined and measured. American Journal of Psychology, 15:201-293, 1904.

[75] M. C. Kidwell, L. B. Lazarević, E. Baranski, T. E. Hardwicke, S. Piechowski, L.-S. Falkenberg, C. Kennett, A. Slowik, C. Sonnleitner, C. Hess-Holden, T. M. Errington, S. Fiedler, and B. A. Nosek. Badges to acknowledge open practices: A simple, low cost, effective method for increasing transparency. PLOS Biology, 14:e1002456, 2016.

[76] B.A. Nosek, G. Alter, G. C. Banks, D. Borsboom, S. D. Bowman, S. J. Breckler, S. Buck, C. D. Chambers, G. Chin, G. Christensen, M. Contestabile, A. Dafoe, E. Eich, J. Freese, R. Glennerster, D. Goroff, D. P. Green, B. Hesse, M. Humphreys, J. Ishiyama, D. Karlan, A. Kraut, A. Lupia, P. Mabry, T. A. Madon, N. Malhotra, E. Mayo-Wilson, M. McNutt, E. Miguel, E. Levy Paluck, U. Simonsohn, C. Soderberg, B. A. Spellman, J. Turitto, G. VandenBos, S. Vazire, E.-J. Wagenmakers, R. Wilson, and T. Yarkoni. Promoting an open research culture. Science, 348:1422-1425, 2015.

[77] B. Aczel, B. Szaszi, A. Sarafoglou, Z. Kekecs, Š. Kucharský, D. Benjamin, C. D. Chambers, A. Fisher, A. Gelman, M. A. Gernsbacher, J. P. Ioannidis, E. Johnson, K. Jonas, S. Kousta, S. O. Lilienfeld, D. S. Lindsay, C. C. Morey, M. Munafò, B. R. Newell, H. Pashler, D. R. Shanks, D. J. Simons, J. M. Wicherts, D. Albarracin, N. D. Anderson, J. Antonakis, H. Arkes, M. D. Back, G. C. Banks, C. Beevers, A. A. Bennett, W. Bleidorn, T. W. Boyer, C. Cacciari, A. S. Carter, J. Cesario, C. Clifton, R. M. Conroy, M. Cortese, F. Cosci, N. Cowan, J. Crawford, E. A. Crone, J. Curtin, R. Engle, S. Farrell, P. Fearon, M. Fichman, W. Frankenhuis, A. M. Freund, M. G. Gaskell, R. Giner-Sorolla, D. P. Green, R. L. Greene, L. L. Harlow, F. Hoces de la Guardia, D. Isaacowitz, J. Kolodner, D. Lieberman, G. D. Logan, W. B. Mendes, 
L. Moersdorf, B. Nyhan, J. Pollack, C. Sullivan, S. Vazire, and E.-J. Wagenmakers. A consensus-based transparency checklist. Nature Human Behaviour, 4:4-6, 2020.

[78] M. D. Wilkinson, M. Dumontier, IJ. J. Aalbersberg, G. Appleton, M. Axton, A. Baak, N. Blomberg, J.-W. Boiten, L. B. da Silva Santos, P. E. Bourne, J. Bouwman, A. J. Brookes, T. Clark, M. Crosas, I. Dillo, O. Dumon, S. Edmunds, C. T. Evelo, R. Finkers, A. Gonzales-Beltran, A. J. G. Gray, P. Groth, C. Goble, Grethe J. S., J. Heringa, P. A. C. 't Hoen, R. Hooft, T. Kuhn, R. Kok, J. Kok, S. J. Lusher, M. E. Martone, A. Mons, A. L. Packer, B. Persson, P. Rocca-Serra, M. Roos, R. van Schaik, S.-A. Sansone, E. Schultes, T. Sengstag, T. Slater, G. Strawn, M. A. Swertz, M. Thompson, J. van der Lei, E. van Mulligen, J. Velterop, A. Waagmeester, P. Wittenburg, K. Wolstencroft, J. Zhao, and B. Mons. The FAIR Guiding Principles for scientific data management and stewardship. Scientific Data, 3, 2016.

[79] Olivier Klein, Tom E Hardwicke, Frederik Aust, Johannes Breuer, Henrik Danielsson, Alicia Hofelich Mohr, Hans IJzerman, Gustav Nilsonne, Wolf Vanpaemel, and Michael C Frank. A practical guide for transparency in psychological science. Collabra: Psychology, 4:1-15, 2018.

[80] G. Alter and R. Gonzalez. Responsible practices for data sharing. American Psychologist, 73:146-156, 2018.

[81] E.-J. Wagenmakers, S. Kucharsky, and the JASP Team, editors. The JASP Data Library. JASP Publishing, Amsterdam, 2020.

[82] Darren B Taichman, Peush Sahni, Anja Pinborg, Larry Peiperl, Christine Laine, Astrid James, Sung-Tae Hong, Abraham Haileamlak, Laragh Gollogly, Fiona Godlee, Frank A Frizelle, and Fernando Flor. Data sharing statements for clinical trials: A requirement 
of the International Committee of Medical Journal Editors. JAMA, 317:2491-2492, 2017.

[83] IJsbrand Jan Aalbersberg, Tom Appleyard, Sarah Brookhart, Todd Carpenter, Michael Clarke, Stephen Curry, Josh Dahl, Alexander DeHaven, Eric Eich, Maryrose Franko, Leonard Freedman, Chris Graf, Sean Grant, Brooks Hanson, Heather Joseph, Veronique Kiermer, Bianca Kramer, Alan Kraut, Roshan Kumar Karn, Carole Lee, Aki MacFarlane, Maryann Martone, Evan Mayo-Wilson, Marcia McNutt, Meredith McPhail, David Mellor, David Moher, Alison Mudditt, Brian Nosek, Belinda Orland, Timothy Parker, Mark Parsons, Mark Patterson, Solange Santos, Carolyn Shore, Daniel Simons, Bobbie Spellman, Jeffrey Spies, Matthew Spitzer, Victoria Stodden, Sowmya Swaminathan, Deborah Sweet, Anne Tsui, and Simine Vazire. Making science transparent by default; Introducing the TOP statement. OSF Preprints, 2018. URL https://osf.io/sm78t.

[84] J. H. Bennett, editor. Statistical Inference and Analysis: Selected Correspondence of R. A. Fisher. Clarendon Press, Oxford, 1990. 